\title{
Direct synthesis of dimethyl ether in multi-tubular fixed-bed reactors: 2D multi-scale modelling and optimum design
}

\author{
Raquel Peláez, Pablo Marín, Fernando V. Díez, Salvador Ordóñez* \\ Department of Chemical and Environmental Engineering, University of Oviedo, Faculty of \\ Chemistry, Julián Clavería 8, Oviedo 33006, Asturias, SPAIN.
}

* Corresponding author: Salvador Ordóñez

Phone: 34-985 103 437,FAX: 34-985 103 434, e-mail: sordonez@uniovi.es

\begin{abstract}
The scope of this work is to explore the viability of the direct synthesis of dimethyl ether (DME) over bifunctional catalysts, such as mixtures of $\mathrm{CuO} / \mathrm{ZnO} / \mathrm{Al}_{2} \mathrm{O}_{3}$ and $\gamma-\mathrm{Al}_{2} \mathrm{O}_{3}$ at industrial scale. To accomplish this purpose, the process is simulated using a phenomenological mathematical model considering momentum, mass and energy balances, applied to both the catalyst particles and reactor bed, which is solved in 2D axi-symmetric coordinates. This constitutes a step beyond most of the available studies for the modelling of the DME synthesis reaction, based on simple 1D isothermal models.

The use of this detailed model revealed the importance of intraparticle mass and heat transfer, with effectiveness factors within the range 0.5-1.1. At the reactor scale, radial phenomena were found to be relevant. A design-sensitivity analysis of mass flux, catalyst fraction, pressure, feed temperature, cooling potential and tube diameter on the reactor performance was carried out. An optimized reactor design that provides $80 \%$ CO conversion operating at inlet temperature and pressure $245^{\circ} \mathrm{C}$ and 40 bar, corresponds to $0.02 \mathrm{~m}$ diameter, $8.50 \mathrm{~m}$ length and $3600 \mathrm{~h}^{-1}$ gas-hourly space velocity with a yield of dimethyl ether of 0.53 .
\end{abstract}

Keywords: synthetic fuels, process intensification, hybrid catalysts, phenomenological modeling, multi-scale modeling. 


\section{Introduction}

Industrial relevance of dimethyl ether (DME) has largely increased in the last years because it is environmentally friendly, has a wide range of applications, and can be manufactured from renewable resources. DME can be used directly as fuel, replacing LPG and conventional diesel fuel, with better combustion performance (lower gasses and soot emissions) [1], or as a fuel precursor and bioplatform molecule for the manufacture of other chemicals of interest, such as olefins, methyl acetate, formaldehyde or dimethyl sulfate, among others [25]. DME production has also an important role in the context of $\mathrm{CO}_{2}$ valorization technologies. DME synthesis from $\mathrm{CO}_{2}$-rich feeds could be an attractive route to recycle the greenhouse gas $\mathrm{CO}_{2}$ by converting it into valuable products and thereby controlling its emission into the atmosphere [6]. Innovative reactor configurations, such as membrane reactors, are being developed to improve this reaction, typically with lower conversion and DME yield compared with the reaction from syngas $[7,8]$.

The most mature route for DME synthesis from syngas consists of a two-step process: in a first reactor, methanol is synthesized in the presence of a metal catalyst, and in a second reactor methanol is dehydrated to DME over a solid acid catalyst. This manufacturing method requires high operation pressures, since methanol synthesis is limited by equilibrium. In order to overcome this limitation, a new one-step synthesis process has been proposed and developed using bifunctional catalysts in a single reactor, with promising results, e.g. CO conversion in a direct DME synthesis reactor can be up to $80 \%$, while a methanol synthesis reactor reaches only $20 \%$ [9].

Many of the published studies are focused in the development of new bifunctional or hybrid catalysts, being $\mathrm{CuO} / \mathrm{ZnO} / \mathrm{Al}_{2} \mathrm{O}_{3}$ and $\gamma-\mathrm{Al}_{2} \mathrm{O}_{3}$ the catalysts most commonly used as metal and acid functions, respectively. Several preparation methods have been studied, although physical mixing of both components seems to be the most suitable [10].

An important issue to be considered in direct DME synthesis is the reactor selection. Lu et al. [11] performed a comparison between fixed bed, fluidized bed and slurry reactors for this process, finding that fluidized beds are the best choice. However, this technology is not proven beyond small pilot plant scale, so its industrial suitability has not been demonstrated yet. Slurry reactors show slow mass transfer of reactants to the catalyst, and low solubility 
and diffusivity of reactants in the liquid phase. Therefore, the reactor type selected in the present work is the fixed bed reactor, in the form of cooled shell and tube, or sequences of adiabatic beds with intercooling [12].

Optimization of the operating conditions to increase DME yield and avoid paraffins formation is a key factor to increase the reaction performance. Another important factor is the formation of hot spots within the catalytic bed due to the large reaction exothermicity, this effect leading to irreversible catalyst deactivation. As the catalyst behavior is strongly influenced by the reaction conditions, the development of a kinetic model for describing the system is essential to model and optimize the process. There are many studies on the kinetics of the integrated process, but most of them are based on kinetic equations obtained separately for the two involved reactions, methanol synthesis and methanol dehydration [810]. In a previous work of our group, an original kinetic model based on the simultaneous reactions involved in the process, working with a physical mixture of commercial $\mathrm{CuO} / \mathrm{ZnO} / \mathrm{Al}_{2} \mathrm{O}_{3}$ and $\gamma-\mathrm{Al}_{2} \mathrm{O}_{3}$ catalysts in a fixed bed reactor, was proposed and experimentally validated [13]. This model predicts the reactor behavior at a laboratory scale, with small catalyst particles, where mass and heat transport limitations are negligible. However, this is not necessarily true for industrial reactors. So, this model must be extended in order to be used for predicting the behavior of industrial reactors. The extended model should consider the spatial distribution of the catalyst components, and provide accurate information about temperature and composition profiles in the catalyst bed to allow the control of the process.

Some works on simulation of the direct synthesis process are available in the literature. Based on the process developed by KOGAS, Kim et al. [14] simulated a DME demonstration plant with Aspen Plus and used the results to propose modification of the process, but give very little details on the process conditions. McBride et al. [15] also simulated the process of DME synthesis in a fixed bed reactor using a layered arrangement of the two catalysts, finding that the physical mixtures of the catalysts gave better results. Vakili et al. [16] developed an steady-state one-dimensional heterogeneous model for a process consisting of two fixed bed reactors in series. The model was used to obtain the optimal reactors configuration and operating conditions. Chen et al. [17] simulated the whole production process, considering the reactions at equilibrium, using Aspen Plus, and studied the energy 
integration through the pinch technology using the program SuperTarget. Other study was performed by Manenti et al. [18], applying a systematic staging design to a system of two fixed-bed reactors in series used for methanol and DME synthesis, and performing a modelbased integrated energy process optimization. They found that the integration of DME production with methanol synthesis increases notably the efficiency of the process. De Falco et al. [19] centered their simulation studies on the possibility of using $\mathrm{CO}_{2}$ rich feeds for the DME direct synthesis, finding that water removal during $\mathrm{CO}_{2}$ conversion is necessary.

Lee et al. [12] proposed a comprehensive one-dimensional steady-state model for the catalytic heterogeneous catalyst bed, considering heat and mass transfer between the catalyst pellets and reactants, and the effectiveness factor of the catalyst, together with the reactor cooling through the reactor wall. They compared a physical mixture of the 2 catalyst pellets and a hybrid catalyst. They found that the hybrid catalyst gave better CO conversion and DME productivity, but reactor temperature control was more difficult. They also reported strong pore diffusion effects. Song et al. [20] used a similar model, and compared simulation results with the obtained for a pilot reactor with good results. They found that the calculated effectiveness factor for the catalyst particles varied markedly along the reactor, and had unusual values. All these studies used 1D-models, where variations in the reactor radial coordinate are not considered, and then some potentially relevant mass and heat transfer phenomena involved are neglected.

The scope of this work is the development of a more accurate model for the direct synthesis of DME from syngas in a fixed bed reactor. The model proposed here is $2 D$, so that the temperature and composition gradients are considered in both axial and radial coordinates at two scales: particle scale and reactor scale. Based on this model, a design-sensitivity analysis can be performed, in order to explore the optimal conditions for the reaction, in terms of relevant variables, such as catalysts fraction, pressure, feed temperature, tube diameter, mass flux and cooling potential. 


\section{Methodology}

\subsection{Modelling: catalyst particle}

The first step in the development of our model for the fixed-bed catalytic reactor is the modelling of the reaction at the particle scale. The kinetics of the reaction of synthesis of dimethyl ether from syngas using simultaneously $\mathrm{CuO} / \mathrm{ZnO} / \mathrm{Al}_{2} \mathrm{O}_{3}$ and $\mathrm{Al}_{2} \mathrm{O}_{3}$ catalysts was experimentally studied in a previous work [13] at a laboratory scale reactor in the absence of mass and heat transfer limitations (using very small catalyst particles). The process can be modelled according to a four reactions scheme, as summarized in Table 1 . The rate equations were proposed based on the mechanistic model of [21] and [22]. Some equations include a term (called here DEN) to account for the inhibition due to water competitive adsorption. The water-gas-shift reaction was found to be in equilibrium.

Table 1. Kinetic model for the synthesis of dimethyl ether from syngas on $\mathrm{CuO} / \mathrm{ZnO} / \mathrm{Al}_{2} \mathrm{O}_{3}$ (catalyst 1) and $\mathrm{Al}_{2} \mathrm{O}_{3}$ (catalyst 2) [13].

\begin{tabular}{|c|c|c|}
\hline Catalyst & Reaction & Rate law \\
\hline \multirow[t]{4}{*}{ Cat 1} & $\begin{array}{c}{[\mathrm{I}] \mathrm{CO}+2 \mathrm{H}_{2} \rightleftarrows \mathrm{CH}_{3} \mathrm{OH}} \\
\Delta H_{1}=-90.51 \mathrm{~kJ} / \mathrm{mol}\end{array}$ & $\begin{array}{l}r_{1}=\frac{k_{1}}{\mathrm{DEN}^{3}}\left(f_{C O} f_{\mathrm{H}_{2}}-\frac{f_{\mathrm{CH}_{3} \mathrm{OH}}}{K_{e q 1} f_{\mathrm{H}_{2}}}\right) \\
\ln k_{1}=25.25-20664 / T\end{array}$ \\
\hline & 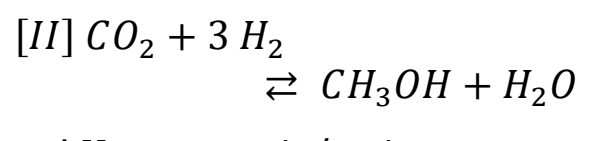 & $r_{2}=\frac{k_{2}}{\mathrm{DEN}^{3}}\left(f_{\mathrm{CO}_{2}} f_{\mathrm{H}_{2}}-\frac{f_{\mathrm{CH}_{3} \mathrm{OH}} f_{\mathrm{H}_{2} \mathrm{O}}}{K_{e q 2} f_{\mathrm{H}_{2}}^{2}}\right)$ \\
\hline & $\Delta H_{2}=-49.51 \mathrm{~kJ} / \mathrm{mol}$ & $\ln k_{2}=-5.10-457 / T$ \\
\hline & $\begin{array}{l}{[I I I] \mathrm{CO}+\mathrm{H}_{2} \mathrm{O} \underset{\mathrm{C}}{\rightleftarrows} \mathrm{CO}_{2}+\mathrm{H}_{2}} \\
\quad \Delta \mathrm{H}_{3}=-41 \mathrm{~kJ} / \mathrm{mol}\end{array}$ & $r_{3}=k_{3}\left(f_{C O} f_{\mathrm{H}_{2} \mathrm{O}}-\frac{f_{\mathrm{CO}_{2}} f_{\mathrm{H}_{2}}}{K_{e q 3}}\right)$ \\
\hline \multirow{3}{*}{ Cat 2} & {$[\mathrm{IV}] 2 \mathrm{CH}_{3} \mathrm{OH} \rightleftarrows \mathrm{C}_{2} \mathrm{H}_{6} \mathrm{O}+\mathrm{H}_{2} \mathrm{O}$} & $r_{4}=k_{4}\left(f_{\mathrm{CH}_{3} \mathrm{OH}}^{2}-\frac{f_{\mathrm{C}_{2} \mathrm{H}_{6} \mathrm{O}} f_{\mathrm{H}_{2} \mathrm{O}}}{K}\right)$ \\
\hline & $\Delta H_{4}=-23.5 \mathrm{~kJ} / \mathrm{mol}$ & \\
\hline & & $\mathrm{DEN}=1+K_{\mathrm{H}_{2} \mathrm{O}} f_{\mathrm{H}_{2} \mathrm{O}}, K_{\mathrm{H}_{2} \mathrm{O}}=19 \mathrm{bar}^{-1}$ \\
\hline
\end{tabular}

${ }^{*} r_{j}$ in $\mathrm{mol} \mathrm{kg}_{\mathrm{cat}}{ }^{-1} \mathrm{~s}^{-1}, f_{i}$ in bar and $\mathrm{T}$ in $\mathrm{K}$. 
The fugacities $\left(f_{i}\right)$ were calculated from the corresponding fugacity coefficients, which were determined according to the literature [23] using the virial equation of state (Tsonopoulus method, [24]). The equilibrium constants were calculated using the temperature-dependent expressions provided by [25].

At industrial scale, catalysts are used in fixed beds shaped as large particles, e.g. as pellets or monoliths. Thus, mass and heat transport inside the catalyst influence the reaction rate. In order to take into account these transport effects, the diffusion-reaction model of Table 2 has been used [26]. In our case, the catalyst is supposed to be shaped as cylindrical pellets (very common in industrial practice), so the model is solved in a 2D axisymmetric coordinate system with gradients in the axial $(z)$ and radial $(r)$ coordinates. The transport from the catalyst surface to the bulk gas is accounted for in the boundary conditions and modelled using the film theory.

Table 2. Particle model: 2D axisymetric diffussion-reaction steady state model.

\begin{tabular}{ll}
\hline Mass balance & Boundary conditions \\
\hline$-\nabla \cdot\left(D_{S i} \nabla c_{i}\right)=\rho_{S} \sum_{j} v_{i j} r_{j}, \quad i=1 \ldots N$ & $\begin{array}{l}\left.\left(-D_{S i} \nabla c_{i}\right)\right|_{\text {surface }}=K_{G i}\left(c_{i b}-c_{i}\right) \\
\left.\left(-D_{S i} \nabla c_{i}\right)\right|_{r_{P}=0}=0\end{array}$ \\
\hline Energy balance & Boundary conditions \\
\hline$-\nabla \cdot\left(k_{S} \nabla T_{S}\right)=\rho_{S} \sum_{j}\left(-\Delta H_{j}\right) r_{j}$ & $\left.\left(-k_{S} \nabla T_{S}\right)\right|_{\text {surface }}=h\left(T_{b}-T_{S}\right)$ \\
& $\left.\left(-k_{S} \nabla T_{S}\right)\right|_{r_{P}=0}=0$ \\
\hline
\end{tabular}

The geometrical and physical properties of the two catalysts present in the reactor required to solve the particle model, are summarized in Table 3. These properties were experimentally determined (particles dimensions and density) or using standard characterization methods (surface area analysis by nitrogen physisorption) [13]. The effective diffusion inside the catalyst pellets $\left(D_{S i}\right)$ was estimated as the contribution of molecular $\left(D_{i m}\right)$ and Knudsen $\left(D_{K i}\right)$ diffusion mechanisms, by the following expression: $D_{S i}=\left(\epsilon_{i n t} / \tau_{c}\right) /\left(1 / D_{i m}+1 / D_{K i}\right)$. The diffusion coefficients, $D_{i m}$ and $D_{K i}$, were calculated using expressions from the literature [24]. Catalyst tortuosity was estimated as $\tau_{c}=1 / \epsilon_{\text {int }}$. 
In the reaction term, $r_{j}$ is the rate of reaction $j$ over catalyst 1 or 2 (in $\mathrm{mol} / \mathrm{kg}_{\text {cat }} \mathrm{s}$ ) calculated with the expressions of Table 1 and $v_{i j}$ are the stoichiometric coefficients of compound $i$ in reaction $j$.

The film mass $\left(K_{G i}\right)$ and heat $(h)$ transport coefficients were calculated using the correlations in Table 4.

Table 3. Geometrical and physical properties of the catalysts.

\begin{tabular}{lll}
\hline Property & Catalyst 1 & Catalyst 2 \\
\hline Catalyst formulation & $\mathrm{CuO} / \mathrm{ZnO} / \mathrm{Al}_{2} \mathrm{O}_{3}$ & $\gamma-\mathrm{Al}_{2} \mathrm{O}_{3}$ \\
Particle diameter $\left(d_{P}\right), \mathrm{mm}$ & 5 & 4.4 \\
Particle length, $\mathrm{mm}$ & 4.79 & 9.14 \\
Equivalent diameter* $\left(d_{e}\right), \mathrm{mm}$ & 2.82 & 3.21 \\
Sphericity $(\phi)$ & 0.873 & 0.828 \\
Specific surface $\left(a_{P}\right), \mathrm{m}^{2} / \mathrm{m}^{3}$ & 2470 & 1784 \\
Solid density $\left(\rho_{S}\right), \mathrm{kg} / \mathrm{m}^{3}$ & 2366 & 1060 \\
Thermal conductivity $\left(k_{S}\right), \mathrm{W} / \mathrm{m} \mathrm{K}$ & 1 & 1 \\
Pore size (nm) & 11.4 & 6.4 \\
Internal porosity $\left(\epsilon_{\text {int }}\right)$ & 0.608 & 0.581 \\
Internal tortuosity $\left(\tau_{c}\right)$ & 1.64 & 1.72 \\
\hline
\end{tabular}

* diameter of the sphere with the same volume

The particle model was solved by the Finite Element Method (FEM) using COMSOL Multiphysics software and finer-predefined free triangular mesh with 986 elements and PARDISO solver.

\subsection{Modelling: reactor}

The reactor considered in this work for the direct synthesis of dimethyl ether consists of a multi-tubular fixed-bed. For simplicity, only one tube is considered for modelling; the rest are assumed to have the same performance (i.e. geometry, feed refrigeration, etc. are the same for all the tubes of the reactor). 
Table 4. Bed properties for the reactor model.

\begin{tabular}{|c|c|c|}
\hline \multicolumn{2}{|l|}{ Bed properties } & \multirow{3}{*}{$\begin{array}{l}\text { Refs. } \\
{[27]}\end{array}$} \\
\hline Bed porosity & $\epsilon_{b}=\epsilon_{b 0}+\left(1-\epsilon_{b 0}\right) \exp \left(-\frac{8}{d_{e}}\left(\frac{D_{R}}{2}-r\right)\right)$ & \\
\hline & With $\epsilon_{b 0}=0.36$ & \\
\hline Permeability & $\kappa=\frac{\epsilon_{b}^{3} \phi^{2} d_{e}^{2}}{150\left(1-\epsilon_{b}\right)^{2}}$ & [27] \\
\hline Forchheimer drag & $\beta_{F}=\frac{1.75\left(1-\epsilon_{b}\right) \rho_{G}}{\epsilon_{b}^{3} \phi d_{e}}$ & {$[27]$} \\
\hline \multirow[t]{2}{*}{$\begin{array}{l}\text { Bed effective } \\
\text { dispersion coefficient }\end{array}$} & $D_{i e z}=\left(1-\sqrt{1-\epsilon_{b}}\right) D_{i m}+\frac{|\boldsymbol{u}| d_{P}}{2}$ & {$[26]$} \\
\hline & $D_{i e r}=\left(1-\sqrt{1-\epsilon_{b}}\right) D_{i m}+\frac{|\boldsymbol{u}| d_{P}}{8}$ & {$[26]$} \\
\hline $\begin{array}{l}\text { Gas-to-solid mass } \\
\text { transfer coefficient }\end{array}$ & $K_{G i}=\frac{D_{i m}}{d_{P}}\left(2+1.1 R e^{0.6} S c_{i}^{1 / 3}\right)$ & {$[28,29]$} \\
\hline \multirow[t]{3}{*}{$\begin{array}{l}\text { Bed effective thermal } \\
\text { conductivity }\end{array}$} & $k_{e z}=k_{b 0}+k_{G}\left(\frac{R e P r}{1.4}\right)$ & {$[26]$} \\
\hline & $k_{e r}=k_{b 0}+k_{G}\left(\frac{\operatorname{RePr}}{8.65\left[1+19.4\left(d_{P} / D_{R}\right)^{2}\right]}\right)$ & {$[26]$} \\
\hline & $k_{b 0}=k_{G}\left(\frac{k_{S}}{k_{G}}\right)^{\left[0.28-0.757 \log \left(\epsilon_{b}\right)-0.057 \log \left(\frac{k_{S}}{k_{G}}\right)\right]}$ & {$[26]$} \\
\hline $\begin{array}{l}\text { Gas-to-solid heat } \\
\text { transfer coefficient }\end{array}$ & $h=\frac{k_{G}}{d_{P}}\left(2+1.1 \operatorname{Re}^{0.6} \operatorname{Pr}^{1 / 3}\right)$ & {$[28]$} \\
\hline
\end{tabular}

The model for the reactor tube is phenomenological, e.g. based on conservation equations, as shown in Table $5[27,30]$. Due to the exothermal reactions taking place and the heat transferred through the reactor wall, temperature and concentration gradients in the axial and radial coordinates of the reactor tube are expected. Hence, the model equations were solved in a 2D axisymmetric coordinate system. 
Table 5. Reactor model: 2D axisymmetric steady state.

Momentum balance (Brinkman equation)

$\nabla \cdot\left[-p I+\frac{\mu}{\epsilon_{b}}\left(\nabla \boldsymbol{u}+(\nabla \boldsymbol{u})^{T}\right)-\frac{2 \mu}{3 \epsilon_{b}}(\nabla \cdot \boldsymbol{u}) I\right]-\left(\frac{\mu}{\kappa}+\beta_{F}|\boldsymbol{u}|\right) \boldsymbol{u}=0$

Continuity equation: $\nabla\left(\rho_{G} \boldsymbol{u}\right)=0$

Boundary conditions: $\left.u\right|_{\text {inlet }}=u \rho_{G} / \rho_{G 0},\left.\quad p\right|_{\text {outlet }}=p_{\text {ref }}$

Mass balance

$-\rho_{G}(\boldsymbol{u} \cdot \nabla) w_{i}+\nabla \cdot\left(D_{i e} \nabla w_{i}\right)+R_{i}=0, \quad D_{i e}=\left[\begin{array}{cc}D_{i e r} & 0 \\ 0 & D_{i e z}\end{array}\right], \quad i=1 \ldots N$

Source term: $R_{i}=M_{i}\left(1-\epsilon_{b}\right)\left[\left.f_{v} a_{P} K_{G i} c_{G}\left(y_{S i}-y_{i}\right)\right|_{\text {cat } 1}+\left.f_{v} a_{P} K_{G i} c_{G}\left(y_{S i}-y_{i}\right)\right|_{\text {cat } 2}\right]$

Boundary conditions: $\left.w_{i}\right|_{\text {inlet }}=w_{i 0},\left.\quad D_{i e} \nabla w_{i}\right|_{\text {outlet }}=0$

Catalyst 1: $\left.a_{P} K_{G i} c_{G}\left(y_{S i}-y_{i}\right)\right|_{c a t 1}=\rho_{S} \sum_{j=1,2,3} v_{i j} r_{j} \eta_{j}, \quad i=1 \ldots N$

Catalyst 2: $\left.a_{P} K_{G i} c_{G}\left(y_{S i}-y_{i}\right)\right|_{c a t ~}=\rho_{S} \sum_{j=4} v_{i j} r_{j} \eta_{j}, \quad i=1 \ldots N$

Energy balance

$-\rho_{G} C_{P} \boldsymbol{u} \cdot \nabla T+\nabla \cdot\left(k_{e} \nabla T\right)+Q=0, \quad k_{e}=\left[\begin{array}{cc}k_{e r} & 0 \\ 0 & k_{e z}\end{array}\right]$

Source term: $Q=\left(1-\epsilon_{b}\right)\left[\left.f_{v} a_{P} h\left(T_{S}-T\right)\right|_{\text {cat } 1}+\left.f_{v} a_{P} h\left(T_{S}-T\right)\right|_{\text {cat } 2}\right]$

Boundary conditions: $\left.T\right|_{\text {inlet }}=T_{0},\left.T\right|_{\text {wall }}=T_{w},\left.\quad k_{e} \nabla T\right|_{\text {outlet }}=0$

Catalyst 1: $\left.a_{P} h\left(T_{S}-T\right)\right|_{\text {cat } 1}=\rho_{S} \sum_{j=1,2,3}\left(-\Delta H_{j}\right) r_{j} \eta_{j}$

Catalyst 2: $\left.a_{P} h\left(T_{S}-T\right)\right|_{c a t 2}=\rho_{S} \sum_{j=4}\left(-\Delta H_{j}\right) r_{j} \eta_{j}$

Brinkman equation is valid to model flow in porous media, for which it includes permeability $(\kappa)$ and Forchheimer drag $\left(\beta_{F}\right)$ terms [27]. This equation is very useful to model non-ideal flow in beds with low reactor to particle diameter ratio $\left(D_{R} / d_{P}<10\right)$. In these situations, a higher bed porosity in the vicinity of the reactor wall results in higher gas velocity there, which affects both reactor conversion and heat transfer through the reactor wall. To account for this effect, Brinkman equation is solved using an appropriate expression for the radial porosity distribution (see Table 4) [26, 31].

Mass and heat balances include (in this order) convection, dispersion, and source (reaction) terms. Dispersion (mass and heat) in fixed beds is an anisotropic property with higher 
dispersion in the axial coordinate (the main coordinate of the flow) than in the radial one, as shown in Table 4. The source terms account for transport (mass and heat) and reaction in two different types of catalyst particles. So, the fraction of the two catalysts in the reactor ( $f_{v}$ or volume fraction) is considered. The internal surface of the reactor wall is considered to be at constant temperature $T_{w}$. This is a good approximation for the case when the reactor is cooled by a boiling liquid (e.g. water) outside the tubes. The boiling temperature is determined by the pressure outside the tubes, and the heat transfer resistances in the outer side of the tube and the tube wall are very small compared to the resistance inside the tube. The model is completed with the balances to the catalysts particles that include the corresponding reaction terms. The intraparticle transport is accounted for using effectiveness factors calculated using the particle model, explained in Section 2.1.

Physical properties of the gas, such as viscosity, heat capacity or thermal conductivity were calculated using correlations from the literature [24], and gas density using the virial equation of state (Tsonopoulus method, [24]).

The reactor model was solved by the Finite Element Method (FEM) using COMSOL Multiphysics software and mapped mesh with 1030 rectangular elements and MUMPS solver.

\section{Results and discussion}

\subsection{Simulation of mass and heat transfer inside catalyst particles}

Mass and heat transfer inside the catalyst pellets have been studied using the $2 \mathrm{D}$ particle model in order to evaluate the influence of intraparticle transport on the performance of the catalysts pellets. Figure 1 shows the results corresponding to the following representative reaction conditions: bulk gas temperature $T_{b}=260^{\circ} \mathrm{C}$, pressure $p_{\text {ref }}=40$ bar, bulk gas mass flux $u \rho_{G}=6 \mathrm{~kg} / \mathrm{m}^{2} \mathrm{~s}$ and bulk gas composition (mole fraction) $0.475 \mathrm{CO}, 0.475 \mathrm{H}_{2}$ and 0.050 $\mathrm{CO}_{2}$. The radial profiles corresponding to the midpoint of the length of the particle (i.e. 2.4 $\mathrm{mm}$ ) are depicted. As evidenced by these profiles, both gas composition and temperature exhibit important gradients inside the catalyst pellet and, hence, intraparticle resistances cannot be neglected. The compound with the highest gradient is $\mathrm{CO}$, with mole fraction 
decreasing from 0.475 at the surface to 0.335 at the center of the particle. No external relevant mass transfer resistance was found (concentrations at the catalyst surface equal bulk concentrations). For the case of heat transfer, both external and internal heat transfer resistances were found to be relevant (the pellet surface is $3^{\circ} \mathrm{C}$ higher than the bulk, and the center of the particle is $4^{\circ} \mathrm{C}$ higher than the surface).

The information from the simulation can be used to calculate the effectiveness factor $(\eta)$, as the ratio of the average reaction rate in the pellet over the reaction rate at bulk gas conditions. For this case and for reactions 1 and 2, the effectiveness factors are, respectively, $\eta_{1}=1.03$ and $\eta_{2}=1.17$. Effectiveness factors are slightly higher than 1 due to the temperature increase inside the pellet, which overcomes the decrease in reaction rate caused by the decrease in reactants concentration. Song et al. [20], also reported effectiveness factors much higher than 1 for these reactions in some cases, at different conditions. This finding will be studied latter in more detail.

\subsection{Correlation of intraparticle transport}

In order to get detailed quantitative information on the intraparticle transport processes in the direct synthesis of dimethyl ether in the particles of catalysts 1 and 2 at the most common reaction conditions, 324 simulations have been performed, covering the following range of the variables: bulk temperature $T_{b}=250-270^{\circ} \mathrm{C}$, pressure $p_{\text {ref }}=30-50$ bar, mass flux $u \rho_{G}=2-10 \mathrm{~kg} / \mathrm{m}^{2} \mathrm{~s}$ and mole fractions in the bulk gas $\left(y_{C O}=0.062-0.57, y_{H 2}=0.017-\right.$

0.570 and $y_{\mathrm{CO}_{2}}=0.050-0.481$ ). For each set of variables, first, the effectiveness factors of the reactions were calculated from the simulation results. Then, the influence of each studied variable on the effectiveness factors was considered. According to the results obtained, only three variables were found to be statistically significant: bulk gas temperature, pressure and bulk CO mole fraction. Then, the following empirical expressions for the effectiveness factors of reactions 1 and 2 were fitted to the calculated data (in units $\mathrm{K}$ for $T$, bar for $p$ and molar fraction for $\left.y_{c o}\right)$.

$$
\eta_{1}=\exp \left(-1.78+0.0035 T_{b}-0.0025 p-0.98 y_{C o}\right)
$$




$$
\eta_{2}=\exp \left(-1.19+0.0022 T_{b}-0.0021 p-0.41 y_{C o}\right)
$$

Equations 1 and 2 are only valid for the particles and for the range of variables considered. The quality of the fitting is demonstrated in the parity plots depicted in Figure 2 . The effectiveness factors of reactions 1 and 2 vary, respectively, between 0.56 and 1.04 and between 0.67 and 1.05. Within these ranges, the fitted expressions predict the simulation values within $\pm 15 \%$ error. Then, these expressions are very useful for modelling the mass and heat transport processes at the particle scale, and are suitable for incorporation in simulations at the reactor scale.

\subsection{Simulation and sensitivity analysis at the reactor scale}

The influence of the main operation variables on the process performance has been determined using the reactor model, as well as the effectiveness factors developed in previous sections. These expressions take into account the variation of the efficiency factors with temperature, pressure and concentration. As it was already determined, parameters such as mass flux do not affect them, since the resistance to the external mass transfer of the particle is not important. The following variables have been considered in the sensitivity analysis (Table 6): mass flux, catalyst fraction, pressure, feed temperature, cooling potential and tube diameter. The cooling potential is defined as the difference between the inlet temperature of the reactor feed $T_{0}$ and the temperature inside the tube $T_{w}, T_{w}$ being considered as constant in the reactor, as previously explained. These variables have been selected because they are important for both the reaction and for the heat transfer, which is critical for highly exothermic reactions. The other parameters have been maintained constant during this study. For example, the feed composition was set to mole fractions of 0.473 for $\mathrm{CO}$ and $\mathrm{H}_{2}$ and 0.054 for $\mathrm{CO}_{2}$. This composition was found to provide optimum reaction rates in the simulations of the previous section and also experimentally in previous works [13]. 
Table 6. Variables of the sensitivity study.

\begin{tabular}{llll}
\hline Variables & Range & Base & Recommended \\
\hline Mass flux $\left(u \rho_{G}\right)$ & $1-5 \mathrm{~kg} / \mathrm{m}^{2} \mathrm{~s}$ & $2 \mathrm{~kg} / \mathrm{m}^{2} \mathrm{~s}$ & $4 \mathrm{~kg} / \mathrm{m}^{2} \mathrm{~s}$ \\
Catalyst fraction $\left(f_{\text {cat }}\right)$ & $60-90 \mathrm{wt} \%$ & $70 \mathrm{wt} . \%$ & $70 \mathrm{wt} . \%$ \\
Pressure $\left(p_{\text {ref }}\right)$ & $30-50 \mathrm{bar}$ & $40 \mathrm{bar}$ & $40 \mathrm{bar}$ \\
Feed temperature $\left(T_{0}\right)$ & $245-260^{\circ} \mathrm{C}$ & $250^{\circ} \mathrm{C}$ & $245^{\circ} \mathrm{C}$ \\
Cooling potential $\left(T_{0}-T_{w}\right)$ & $0-20^{\circ} \mathrm{C}$ & $0^{\circ} \mathrm{C}$ & $0^{\circ} \mathrm{C}$ \\
Tube diameter $\left(D_{R}\right)$ & $0.02-0.04 \mathrm{~m}$ & $0.03 \mathrm{~m}$ & $0.02 \mathrm{~m}$ \\
\hline
\end{tabular}

The reactor length required to achieve $80 \%$ conversion at each set of conditions was calculated. The target conversion of $80 \%$ has been chosen because it is high, but lower than the equilibrium conversion for all the operating conditions considered. Results are shown in Figures Figure 3. Parametric study: influence of mass flux (a), fraction of catalyst (b) and pressure (c) on the space time necessary for $80 \%$ CO conversion. and

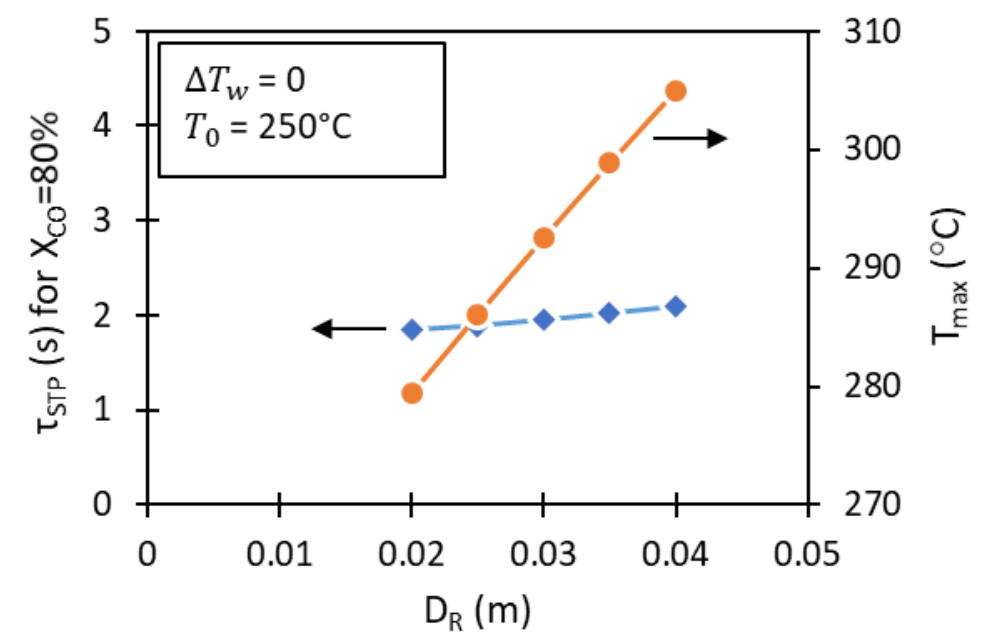

Figure 4. Parametric study: influence of feed temperature (a), wall temperature (b) and reactor diameter (c) on the space time necessary for $80 \%$ CO conversion. in terms of space time, defined as the reactor volume divided by the inlet volumetric flow rate measured at the conditions $\mathrm{T}=298 \mathrm{~K}$ and $\mathrm{p}=1.01 \mathrm{bar}$.

As shown in Figure 3a, space time (and hence, reactor volume per unit of feed), decreases slightly as max flux (and hence gas superficial velocity) increases. Hence, the operation of the reactor at higher mass flux is more efficient. This is explained by the observed (see Figure 3. Parametric study: influence of mass flux (a), fraction of catalyst (b) and pressure (c) on the 
space time necessary for $80 \%$ CO conversion. a) lower maximum temperature attained in the bed, which, in the region close to equilibrium, results in higher reaction rates. Calculated temperature profiles, not shown here, indicate that on increasing mass flux, the hot spot moves in the direction of the flow, and the peak temperature decreases, due to the improved heat transfer to the reactor wall.

The main drawback of operating at high mass flux is the increase in pressure drop (from 2.3 $\mathrm{kPa}$ at $1 \mathrm{~kg} / \mathrm{m}^{2} \mathrm{~s}$ to $40.1 \mathrm{kPa}$ at $\left.5 \mathrm{~kg} / \mathrm{m}^{2} \mathrm{~s}\right)$. This increase is due to both the increase in gas velocity and reactor length. Moreover, at high mass flux $\left(4-5 \mathrm{~kg} / \mathrm{m}^{2} \mathrm{~s}\right)$ the decrease in space time is less pronounced. Taking into account both factors (reactor volume and pressure drop), the value of mass flux more adequate is considered to be in the region of $4 \mathrm{~kg} / \mathrm{m}^{2} \mathrm{~s}$.

The next variable considered was the fraction of each catalyst in the reactor. According to the kinetic experiments, the reactions catalyzed by $\mathrm{CuO} / \mathrm{ZnO} / \mathrm{Al}_{2} \mathrm{O}_{3}$ are slower than the alumina-catalyzed methanol condensation. This fact explains the observed reduction in space time when the fraction of $\mathrm{CuO} / \mathrm{ZnO} / \mathrm{Al}_{2} \mathrm{O}_{3}$ increases (Figure $3 \mathrm{~b}$ ). However, due to the increase in reaction rate, the maximum temperature also increases, and can exceed the maximum temperature limit recommended to prevent catalyst deactivation $\left(280-300^{\circ} \mathrm{C}\right)$. As a consequence, a catalyst fraction around $70 \%$ corresponding to $293^{\circ} \mathrm{C}$ of maximum temperature is recommended.

Pressure is a critical variable in the reaction of synthesis of methanol, because on increasing pressure the reaction equilibrium shifts towards products. However, in the direct synthesis of dimethyl ether, the concomitant synthesis of dimethyl ether from methanol contributes to shift the equilibrium. For this reason, and as shown in Figure $3 c$, an increase in pressure decreases the space time, but not very markedly, particularly above 40 bar: the space time for $80 \%$ conversion decreases $18 \%$ from 30 to 40 bar, but only $5 \%$ from 40 to 50 bar. As for the case of the catalyst fraction, higher pressure results in higher reaction rate and, hence, the maximum temperature increases. A pressure of 40 bar is recommended, corresponding to a maximum reactor temperature of $293^{\circ} \mathrm{C}$. Operating pressures of the direct synthesis of dimethyl ether are lower than for the commercial process of methanol synthesis (50-100 bar) which is an important economic advantage of the direct synthesis of dimethyl ether. 


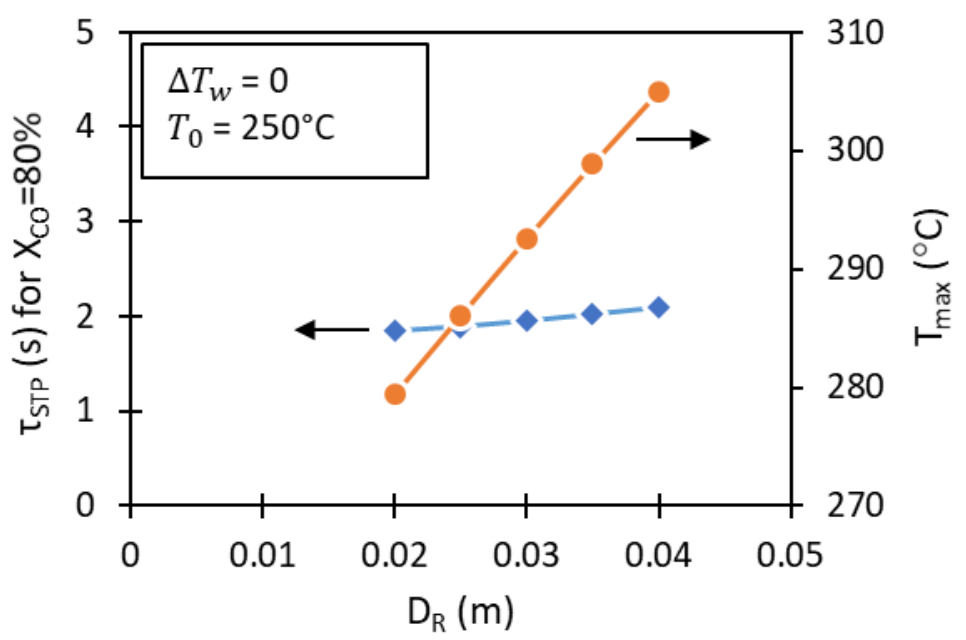

Figure 4 represents the effect of variables related to the thermal behavior of the reactor. The feed temperature was varied in the range $245-260^{\circ} \mathrm{C}$ : an increase in the feed temperature resulted in an increase of both space time needed for $80 \%$ conversion and maximum temperature in the reactor. The maximum reactor temperature increases as the feed temperature increases, as expected. The increase in reactor length is attributed to the resulting decrease of equilibrium conversion, which decreases reaction rate. According to these results, the feed temperature in the range studied is the minimum considered, $245^{\circ} \mathrm{C}$. Note that the rate laws of the reactions were developed for the range $250-270^{\circ} \mathrm{C}$, so feed temperatures below $245^{\circ} \mathrm{C}$ are not considered here.

The effect of cooling temperature was studied using the cooling potential, i.e. the difference between the feed and cooling temperatures. Thus, for a given cooling potential, if the feed temperature changes, the cooling temperature changes accordingly, so the cooling potential is exclusively a measure of the cooling strength. On increasing the cooling potential, both space time and maximum temperature decreased, but the effect is less marked than for the feed temperature. Nevertheless, the cooling potential can be used to control the reactor 
temperature and prevent catalyst overheating. As shown in

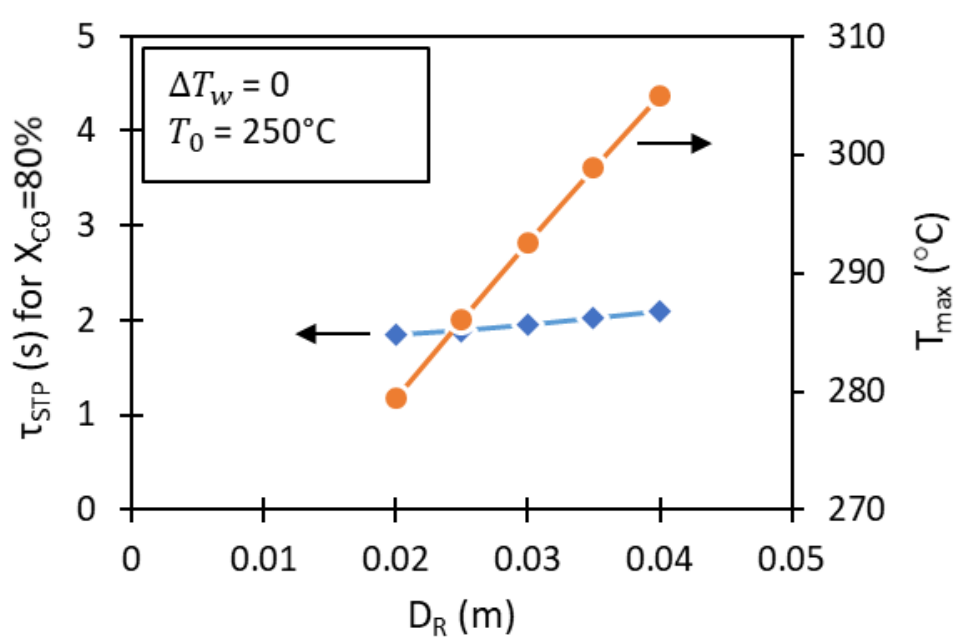

Figure $4 \mathrm{~b}$, the maximum reactor temperature decreases from 293 to $283^{\circ} \mathrm{C}$, when the cooling potential increases from 0 to $20^{\circ} \mathrm{C}$. Hence, the recommendation is to design the reactor considering a cooling potential 0 (e.g. cooling temperature equal to feed temperature) and use the cooling potential as a manipulated variable in the reactor temperature control system.

The last variable considered is the tube diameter, which is critical for the cooling of the

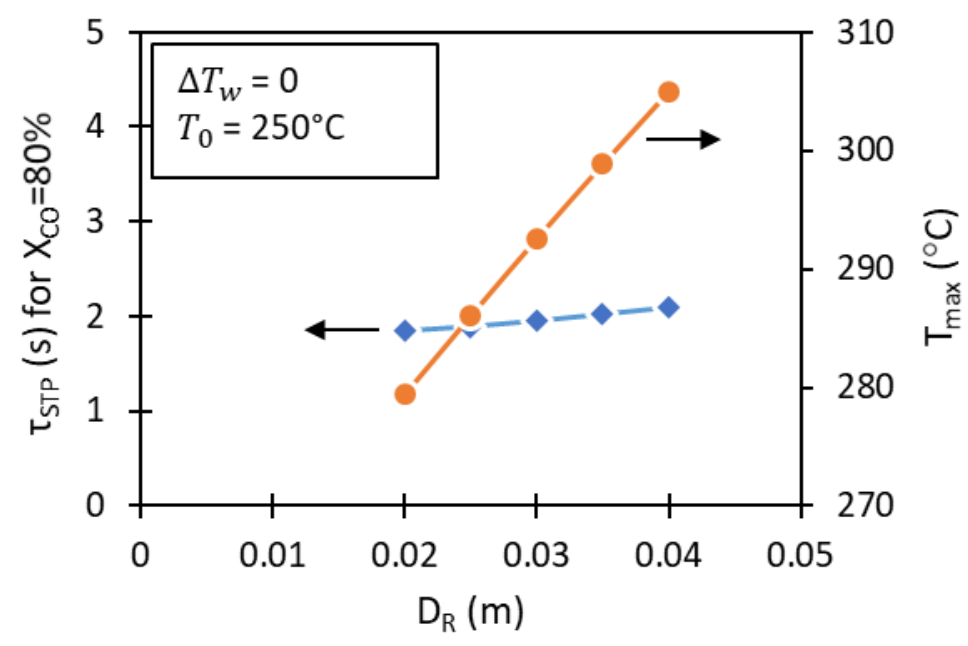

reactor. As observed in

Figure 4c, this variable has a great influence on the maximum temperature. For smaller tube diameters, the reactor surface to volume ratio increases, so heat released by the reactions is transferred at a higher rate and, hence, maximum temperature decreases. The recommended tube diameter in this case is $0.02 \mathrm{~m}$. Below this value, the ratio of tube diameter to particle size (equivalent particle diameter) is too low $(<6.7)$, and deviations 
from plug flow (channeling in the vicinity of the wall due to higher porosity), that have a negative effect in the performance of the reactor, are severe.

According to these results, the recommended values of the variables studied are the ones summarized in Table 6.

\subsection{Reactor design at optimized conditions}

Once the recommended values of the main variables have been estimated (see Table 6), the reactor model is used to study in detail the reactor characteristics and performance at these conditions. Although these recommended conditions are not fully optimized (an optimization would require studying the reactor performance at more combinations of the relevant variables, together with an economic analysis), it is very likely that they are in the vicinity of the optimal conditions, and the results obtained will provide valuable information on the expected performance of the process. The reactor was then simulated, and the calculated length required to achieve $80 \%$ CO conversion was $8.50 \mathrm{~m}$.

The yield of the different products was the following: dimethyl ether $0.534, \mathrm{CO}_{2} 0.264$ and methanol 0.0087 . The low yield of methanol suggests that the equilibrium of the reaction of synthesis of dimethyl ether is shifted towards products. The yield of $\mathrm{CO}_{2}$ is high because the $\mathrm{CO} / \mathrm{H}_{2}$ ratio of the feed was $\mathrm{CO} / \mathrm{H}_{2}=1$. Thus, the water formed as product in the synthesis of dimethyl ether reacts with $\mathrm{CO}$, present in high amount due to the high $\mathrm{CO} / \mathrm{H}_{2}$ ratio of the feed, and generates $\mathrm{CO}_{2}$ and $\mathrm{H}_{2}$ (reaction 3 of Table 1). Likewise, the $\mathrm{H}_{2}$ produced is consumed in the synthesis of methanol (reaction 1 of Table 1). Overall, part of the $\mathrm{H}_{2}$ required for the process is generated in situ and, as a consequence, no pre-treatment units to adjust the composition of the syngas are required.

The surface plots of temperature inside the reactor tube are depicted in Figure 5, where for comparison, the results for the reactor containing only the metal catalyst (i.e. $f_{\text {cat }}=1$ ) are also included. The simulations reveal the presence of a hot spot close to the reactor inlet. According to this, heat transfer in the reactor should be designed taking into account the formation of the hot spot and the prevention of catalyst overheating (e.g. selection of tube diameter and cooling temperature such as that maximum temperature is lower than 280 - 
$300^{\circ} \mathrm{C}$ ). In the simulation carried out at the recommended conditions, the maximum temperature was $267^{\circ} \mathrm{C}$, well below this limit.

The conversion and temperature axial profiles at the reactor axis $(r=0)$ are shown in Figure $6 \mathrm{a}$ and $6 \mathrm{~b}$. This figure can be helpful to compare the performances when the reactor contains catalysts 1 and 2 , and only catalyst 1 (i.e. $f_{\text {cat }}=1$, so no $\gamma-\mathrm{Al}_{2} \mathrm{O}_{3}$ is present, and methanol is formed as final product). In the second case, conversion increases up to $26.6 \%$ in the first quarter of the reactor (approx. $z=2 \mathrm{~m}$ ) and, then, remains constant at the equilibrium. It should be noted that, even with such a low conversion, the hot spot is slightly higher than that for the case of $70 \%$ (wt.) fraction of catalyst $1\left(272^{\circ} \mathrm{C}\right.$ vs. $\left.267^{\circ} \mathrm{C}\right)$. Likewise, the use of $\gamma-\mathrm{Al}_{2} \mathrm{O}_{3}$ catalyst makes it possible to produce dimethyl ether and shifts the equilibrium of the methanol synthesis reaction. For this reason, in this latter case, conversion increases progressively up to $80 \%$ at the end of the reactor. DME yield also increases gradually, reaching a value of 0.534 at the reactor exit.

Regarding to the heat flux axial profile at the reactor wall $\left(r=D_{R} / 2=0.01 \mathrm{~m}\right)$, Figure $6 c$, it increases up from $135 \mathrm{~W} / \mathrm{m}^{2}$ to $750 \mathrm{~W} / \mathrm{m}^{2}$ in the area close to the reactor inlet and then, it decreases progressively to $82 \mathrm{~W} / \mathrm{m}^{2}$ in the reactor outlet. The point with maximum heat flux is associated with the presence of the hot spot, as it has been demonstrated previously.

Figure 7 shows radial profiles (at $z=L_{R} / 2=4.25 \mathrm{~m}$ ) of key properties and variables that illustrate the relevance of radial phenomena, which are taken into account by our 2D model. The model solves the momentum balance (Brinkmann equation) accounting for the radial porosity distribution of the packed bed. Thus, in the vicinity of the wall, the packing of the catalyst particles is less compact and, hence, the bed porosity is higher (this region is around $20 \%$ of the reactor volume). This affects different reactor characteristics, such as reaction rate or heat transfer. Gas superficial velocity exhibits a remarkable behavior (Figure 7), increasing from $0.12 \mathrm{~m} / \mathrm{s}$ at the reactor center to a maximum of $0.47 \mathrm{~m} / \mathrm{s}$ close to the reactor wall and then decreasing to zero at the wall. Higher gas velocity (lower residence time) with less amount of catalyst results in lower conversion in this part of the bed. This negative factor is overcome, at least partially, by the good radial mass transfer observed at the studied conditions (Table 6). The radial heat transfer coefficient exhibits a similar behavior, as turbulence in the gas phase is the main contribution to both radial heat and 
mass transfer. Thus, the radial heat transfer coefficient is $1.7 \mathrm{~W} / \mathrm{m} \mathrm{K}$ at the center of the bed, reaching a value of $5.3 \mathrm{~W} / \mathrm{m} \mathrm{K}$ at the vicinity of the wall. Radial temperature profiles are relevant. Figure 7. Simulation of the reactor model at the optimized operating conditions $\left(\boldsymbol{u} \boldsymbol{\rho}_{G}=4 \mathrm{~kg} / \mathrm{m}^{2} \mathrm{~s}, \boldsymbol{T}_{\mathbf{0}}=245^{\circ} \mathrm{C}, \boldsymbol{\Delta} \boldsymbol{T}_{\boldsymbol{w}}=0, \boldsymbol{p}_{\mathbf{0}}=40 \mathrm{bar}, \boldsymbol{D}_{\boldsymbol{R}}=0.02 \mathrm{~m}\right.$ and $\left.\boldsymbol{L}_{\boldsymbol{R}}=8.50 \mathrm{~m}\right)$ : radial profiles at $z=L_{R} / 2=4.25 \mathrm{~m}$ of (a) bed porosity and superficial velocity and (b) radial temperature profile and radial thermal conductivity.b shows a difference of $9^{\circ} \mathrm{C}$ between the reactor center and the wall, at the considered reactor length, whereas Figure 5. Simulation of the reactor model at the optimum operating conditions $\left(\boldsymbol{u} \boldsymbol{\rho}_{G}=4 \mathrm{~kg} / \mathrm{m}^{2} \mathrm{~s}, \boldsymbol{T}_{\mathbf{0}}=245^{\circ} \mathrm{C}, \boldsymbol{\Delta} \boldsymbol{T}_{\boldsymbol{w}}\right.$ $=0, \boldsymbol{p}_{0}=40$ bar, $\boldsymbol{D}_{\boldsymbol{R}}=0.02 \mathrm{~m}$ and $\boldsymbol{L}_{\boldsymbol{R}}=8.50 \mathrm{~m}$ ): temperature gradient. Comparison of (a) direct DME synthesis $\left(\boldsymbol{f}_{\boldsymbol{c a t}}=0.70 \mathrm{wt}\right.$.) and (b) methanol synthesis $\left(\boldsymbol{f}_{\boldsymbol{c a t}}=1 \mathrm{wt}\right.$.).a shows that in the region of the hot spot, this difference is much higher (about $22^{\circ} \mathrm{C}$ ). This illustrates the importance of using 2D models for studying this reaction.

\section{Conclusions}

The direct synthesis of dimethyl ether from synthesis gas has been studied by means of detailed simulations of a 2D phenomenological model of catalyst particles and reactor bed. This model improves the one-dimensional models available in literature, providing essential information of the process for industrial reactors design. At the catalyst particle scale, it has been found that intraparticle mass and heat transfer resistances are important and must be taken into account. The simulations carried out over a broad range of operating conditions predicted effectiveness factors for the reactions that lied between 0.5 and 1.1. The calculated values of the effectiveness factor were fit to simple expressions capable of predicting the effectiveness factors within $\pm 15 \%$ error.

The simulations at the reactor scale consisted of a design-sensitivity analysis: reactor length required to achieve $80 \% \mathrm{CO}$ conversion was determined for a set of operating and design variables. The conditions that fulfill this requirement and at the same time maintained the maximum temperature below the safety limit were identified. It can be concluded that an increase in catalyst fraction, pressure, feed temperature and tube diameter increases the maximum temperature, while mass flux and cooling potential have the opposite effect. Mass flux and tube diameter had the strongest effect, so it is advised to carefully select these 
variables during the design of the reactor. For operation at $4 \mathrm{~kg} / \mathrm{m}^{2} \mathrm{~s}$ of mass flux and $0.02 \mathrm{~m}$ of tube diameter, the recommended values of reactor length and gas-hourly space velocity are $8.5 \mathrm{~m}$ and $3600 \mathrm{~h}^{-1}$, respectively. Simulation results show that in this reactor radial phenomena are relevant in hydrodynamics and heat and mass transfer, so that 2D models provide more accurate information than 1D models. Finally, the comparison of this recommended design to a similar methanol synthesis reactor showed that the dimethyl ether reactor presents higher CO conversion and lower hot spot.

\section{Acknowledgements}

This work has been financed by the Spanish Ministry of Economy and Competitiveness (CTQ2014-52956-C3-1-R) and by the Government of the Principality of Asturias (contract FC15-GRUPIN14-078). Raquel Peláez acknowledges the Spanish Ministry of Economy and Competitiveness for the PhD grant that supports her research.

\section{References}

[1] T.A. Semelsberger, R.L. Borup, H.L. Greene, Dimethyl ether (DME) as an alternative fuel, J. Power Sources 156 (2006) 497-511.

[2] G. Cai, Z. Liu, R. Shi, H. Changqing, L. Yang, C. Sun, Y. Chang, Light alkenes from syngas via dimethyl ether, Appl. Catal., A 125 (1995) 29-38.

[3] R. Peláez, P. Marín, S. Ordóñez, Synthesis of formaldehyde from dimethyl ether on aluminasupported molybdenum oxide catalyst, Appl. Catal., A 527 (2016) 137-145.

[4] Y. Liu, K. Murata, M. Inaba, I. Takahara, Synthesis of ethanol from methanol and syngas through an indirect route containing methanol dehydrogenation, DME carbonylation, and methyl acetate hydrogenolysis, Fuel Process. Technol. 110 (2013) 206-213.

[5] P. Cheung, A. Bhan, G.J. Sunley, E. Iglesia, Selective Carbonylation of Dimethyl Ether to Methyl Acetate Catalyzed by Acidic Zeolites, Angew. Chem. Int. Ed. 45 (2006) 1617-1620.

[6] C. Ampelli, S. Perathoner, G. Centi, $\mathrm{CO} 2$ utilization: an enabling element to move to a resourceand energy-efficient chemical and fuel production, 2015.

[7] M. De Falco, M. Capocelli, Chapter 5 - Direct Synthesis of Methanol and Dimethyl Ether From a CO2-Rich Feedstock: Thermodynamic Analysis and Selective Membrane Application A2 - Basile, Angelo, in: F. Dalena (Ed.) Methanol, Elsevier, 2018, pp. 113-128.

[8] N. Diban, A.T. Aguayo, J. Bilbao, A. Urtiaga, I. Ortiz, Membrane Reactors for in Situ Water Removal: A Review of Applications, Ind. Eng. Chem. Res. 52 (2013) 10342-10354.

[9] Z. Azizi, M. Rezaeimanesh, T. Tohidian, M.R. Rahimpour, Dimethyl ether: A review of technologies and production challenges, Chem. Eng. Process. 82 (2014) 150-172.

[10] G. Bozga, I.T. Apan, R.E. Bozga, Dimethyl ether synthesis catalysts, processes and reactors, Recent Pat. Catal. 2 (2013) 68-81.

[11] W.-Z. Lu, L.-H. Teng, W.-D. Xiao, Simulation and experiment study of dimethyl ether synthesis from syngas in a fluidized-bed reactor, Chem. Eng. Sci. 59 (2004) 5455-5464. 
[12] S.B. Lee, W. Cho, D.K. Park, E.S. Yoon, Simulation of fixed bed reactor for dimethyl ether synthesis, Korean J. Chem. Eng. 23 (2006) 522-530.

[13] R. Peláez, P. Marín, S. Ordóñez, Direct synthesis of dimethyl ether from syngas over mechanical mixtures of $\mathrm{CuO} / \mathrm{ZnO} / \mathrm{Al} 2 \mathrm{O} 3$ and $\mathrm{y}-\mathrm{Al} 2 \mathrm{O} 3$ : Process optimization and kinetic modelling, Fuel Process. Technol. 168 (2017) 40-49.

[14] I.H. Kim, B.J. Kang, E.S. Yoon, Design Modification Study on DME direct synthesis technology, Comput. Aided Chem. Eng. 31 (2012) 790-794.

[15] K. McBride, T. Turek, R. Güttel, Direct dimethyl ether synthesis by spatial patterned catalyst arrangement: A modeling and simulation study, AlChE J. 58 (2012) 3468-3473.

[16] R. Vakili, R. Eslamloueyan, Optimal design of an industrial scale dual-type reactor for direct dimethyl ether (DME) production from syngas, Chem. Eng. Process. 62 (2012) 78-88.

[17] H.-J. Chen, C.-W. Fan, C.-S. Yu, Analysis, synthesis, and design of a one-step dimethyl ether production via a thermodynamic approach, Appl. Energy 101 (2013) 449-456.

[18] F. Manenti, A.R. Leon-Garzon, Z. Ravaghi-Ardebili, C. Pirola, Systematic staging design applied to the fixed-bed reactor series for methanol and one-step methanol/dimethyl ether synthesis, Appl. Therm. Eng. 70 (2014) 1228-1237.

[19] M. De Falco, M. Capocelli, G. Centi, Dimethyl ether production from CO2 rich feedstocks in a one-step process: Thermodynamic evaluation and reactor simulation, Chem. Eng. J. 294 (2016) $400-$ 409.

[20] D. Song, W. Cho, G. Lee, D.K. Park, E.S. Yoon, Numerical analysis of a pilot-scale fixed-bed reactor for dimethyl ether (DME) synthesis, Ind. Eng. Chem. Res. 47 (2008) 4553-4559.

[21] K.M.V. Bussche, G.F. Froment, A steady-state kinetic model for methanol synthesis and the water gas shift reaction on a commercial Cu/ZnO/Al2O3 catalyst, J. Catal. 161 (1996) 1-10.

[22] G. Bercic, J. Levec, Catalytic dehydration of methanol to dimethyl ether. Kinetic investigation and reactor simulation, Ind. Eng. Chem. Res. 32 (1993) 2478-2484.

[23] J.M. Smith, H.C.V. Ness, M.M. Abbott, Introduction to chemical engineering thermodinamics, 6th ed., McGraw-Hill, 2001.

[24] D.W. Green, R.H. Perry, Perry's Chemical Engineers' Handbook, 8th ed., McGraw-Hill, 2008.

[25] A.T. Aguayo, J. Ereña, D. Mier, J.M. Arandes, M. Olazar, J. Bilbao, Kinetic modeling of dimethyl ether synthesis in a single step on a CuO- ZnO- Al2O3/Y-Al2O3 catalyst, Ind. Eng. Chem. Res. 46 (2007) 5522-5530.

[26] P. Marín, F.V. Díez, S. Ordóñez, Fixed bed membrane reactors for WGSR-based hydrogen production: Optimisation of modelling approaches and reactor performance, Int. J. Hydrogen Energy 37 (2012) 4997-5010.

[27] P. Marín, Y. Patiño, F.V. Díez, S. Ordóñez, Modelling of hydrogen perm-selective membrane reactors for catalytic methane steam reforming, Int. J. Hydrogen Energy 37 (2012) 18433-18445.

[28] C.R. Thompson, P. Marín, F.V. Díez, S. Ordóñez, Evaluation of the use of ceramic foams as catalyst supports for reverse-flow combustors, Chem. Eng. J. 221 (2013) 44-54.

[29] O. Levenspiel, Chemical Reaction Engineering, 3rd ed., Wiley, 1999.

[30] R. Sanz, J.A. Calles, D. Alique, L. Furones, S. Ordóñez, P. Marín, Hydrogen production in a PorePlated Pd-membrane reactor: Experimental analysis and model validation for the Water Gas Shift reaction, Int. J. Hydrogen Energy 40 (2015) 3472-3484.

[31] P. Marín, C. Hamel, S. Ordóñez, F.V. Díez, E. Tsotsas, A. Seidel-Morgenstern, Analysis of a fluidized bed membrane reactor for butane partial oxidation to maleic anhydride: 2D modelling, Chem. Eng. Sci. 65 (2010) 3538-3548. 


\section{Nomenclature}

\section{Latin symbols}

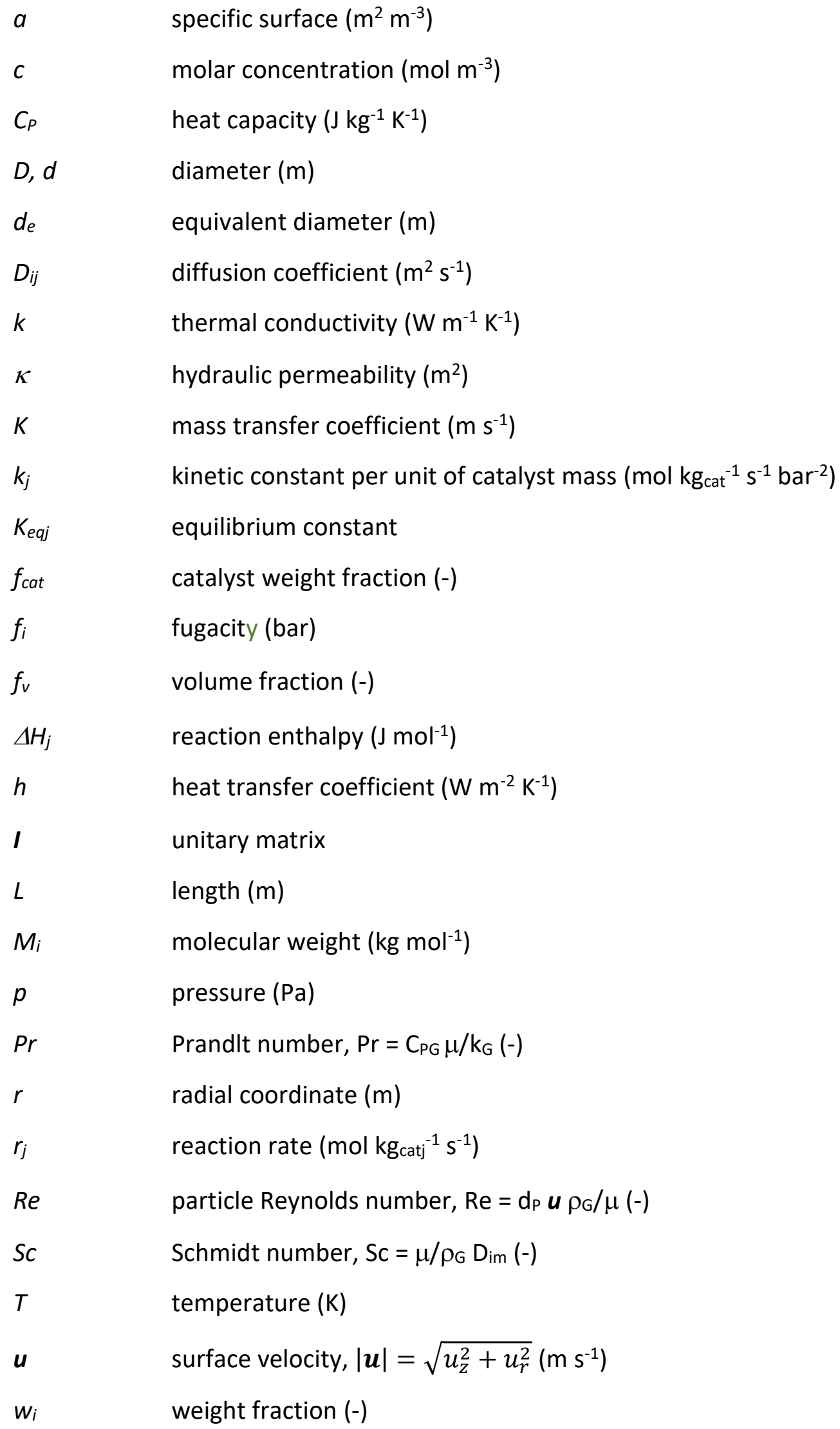




$$
\begin{array}{ll}
y_{i} & \text { molar fraction }(-) \\
z & \text { axial coordinate }(\mathrm{m})
\end{array}
$$

\section{Subscripts/superscripts}

$\begin{array}{ll}0 & \text { inlet } \\ b & \text { bulk } \\ c a t & \text { catalyst } \\ e & \text { effective } \\ G & \text { gas } \\ i & \text { compound } \\ j & \text { reaction } \\ P & \text { particle } \\ r & \text { radial coordinate } \\ R & \text { reactor } \\ r e f & \text { reference conditions } \\ S & \text { solid } \\ S T P & \text { Standard Temperature and Pressure } \\ W & \text { wall } \\ Z & \text { axial coordinate }\end{array}$

Greek symbols

$\beta$

approaching to equilibrium

$\beta_{F} \quad$ Forchheimer drag $\left(\mathrm{kg} \mathrm{m}^{-4}\right)$

$\varepsilon_{b 0} \quad$ average bed porosity (-)

$\varepsilon_{b} \quad$ bed porosity (-)

$\varepsilon_{\text {int }} \quad$ internal porosity (-)

$\phi \quad$ sphericity (-)

$\eta \quad$ effectiveness factor (-)

$\mu \quad$ viscosity $\left(\mathrm{kg} \mathrm{m}^{-1} \mathrm{~s}^{-1}\right)$

$v_{i j} \quad$ stoichiometric coefficient of compound $i$ in reaction $j(-)$

$\rho \quad$ density $\left(\mathrm{kg} \mathrm{m}^{-3}\right)$ 
space time (s)

$\tau_{c}$

catalyst tortuosity (-) 


\section{Figures}

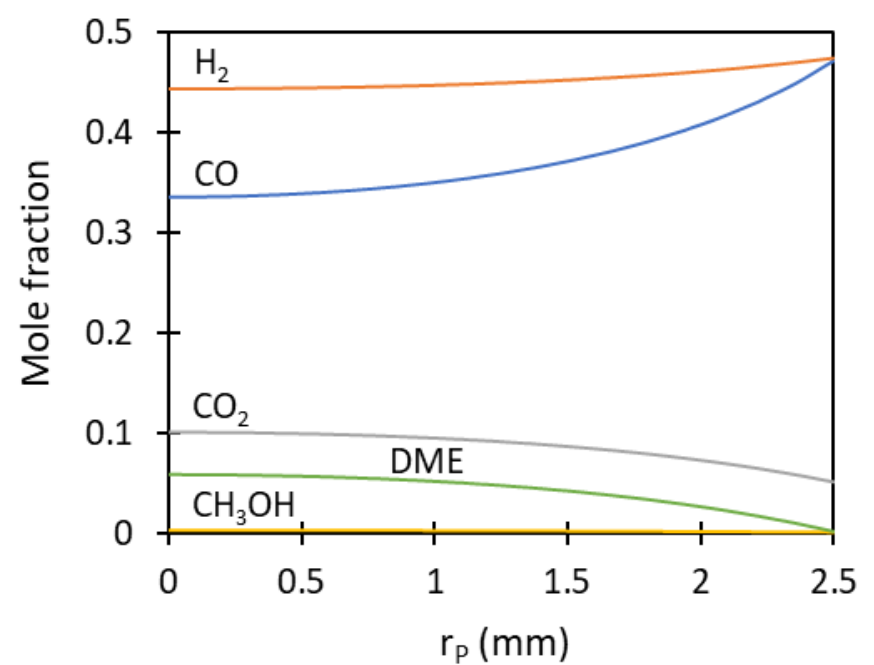

a)

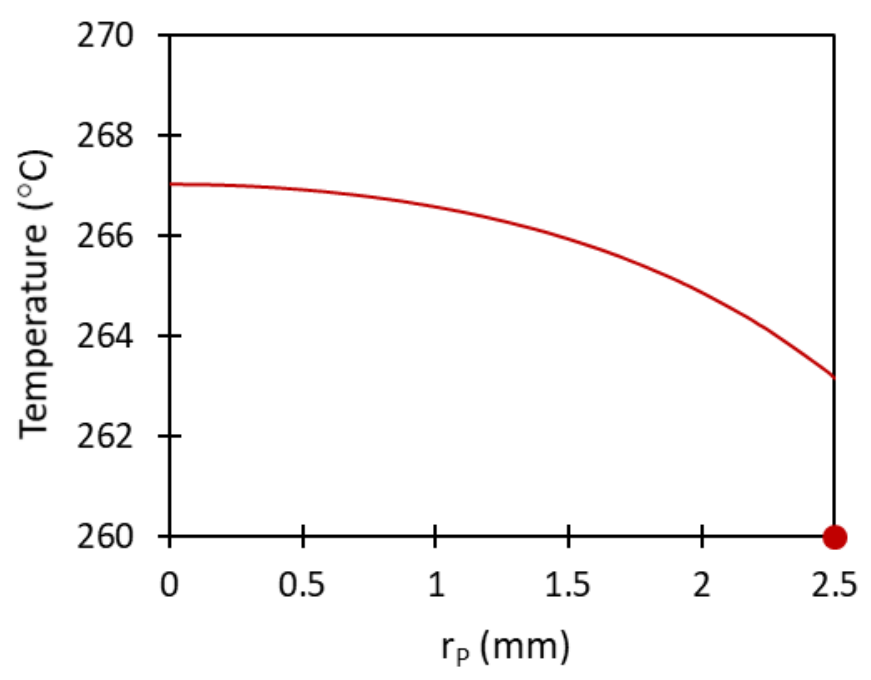

b)

Figure 1. Intraparticle concentration (a) and temperature (b) profiles calculated with the 2D particle model (taken at the half height of the particle). Symbol (๑) indicates the bulk gas temperature. Conditions of the bulk gas: $260^{\circ} \mathrm{C}, 40 \mathrm{bar}, 6 \mathrm{~kg} / \mathrm{m}^{2} \mathrm{~s}$, mole fractions $0.475 \mathrm{CO}$, $0.475 \mathrm{H}_{2}$ and $0.050 \mathrm{CO}_{2} \cdot \boldsymbol{r}_{\boldsymbol{p}}=\mathbf{0}$ corresponds to the centre of the particle. Calculated effectiveness factors: $\boldsymbol{\eta}_{\mathbf{1}} 1.03$ and $\boldsymbol{\eta}_{\mathbf{2}}=1.17$. 


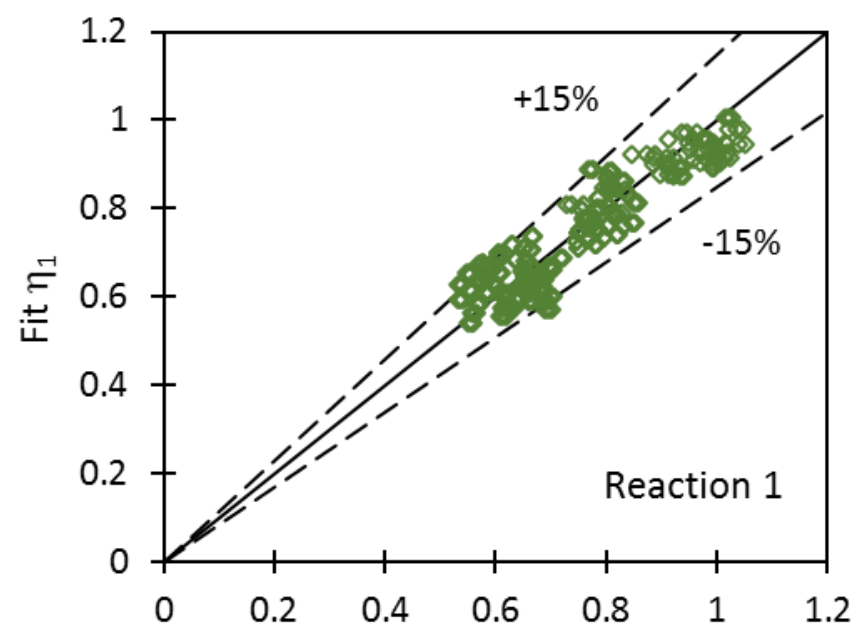

a)

$\eta_{1}$ (2D particle model)

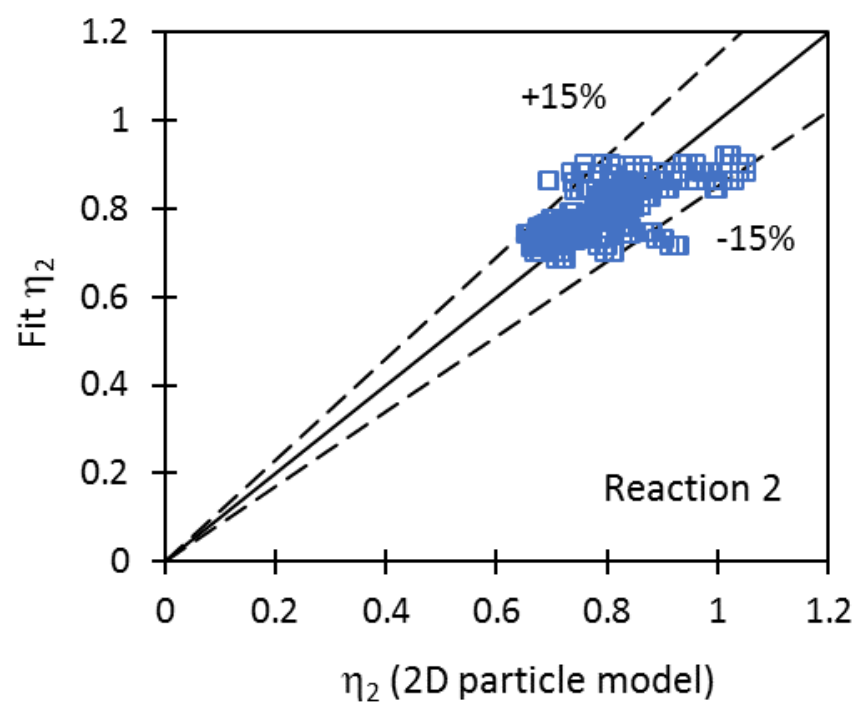

Figure 2. Parity plots for the fitting of the intraparticle effectiveness factors of reactions 1 (a) and 2 (b). Dashed lines indicate the $\pm 15 \%$ error. 


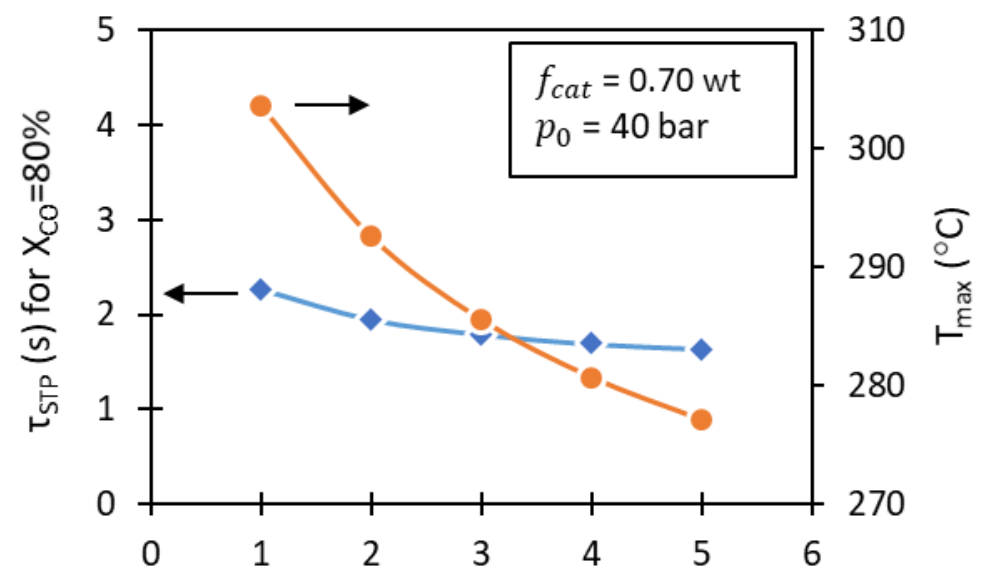

a) $u \rho_{G}\left(\mathrm{~kg} / \mathrm{m}^{2} \mathrm{~s}\right)$

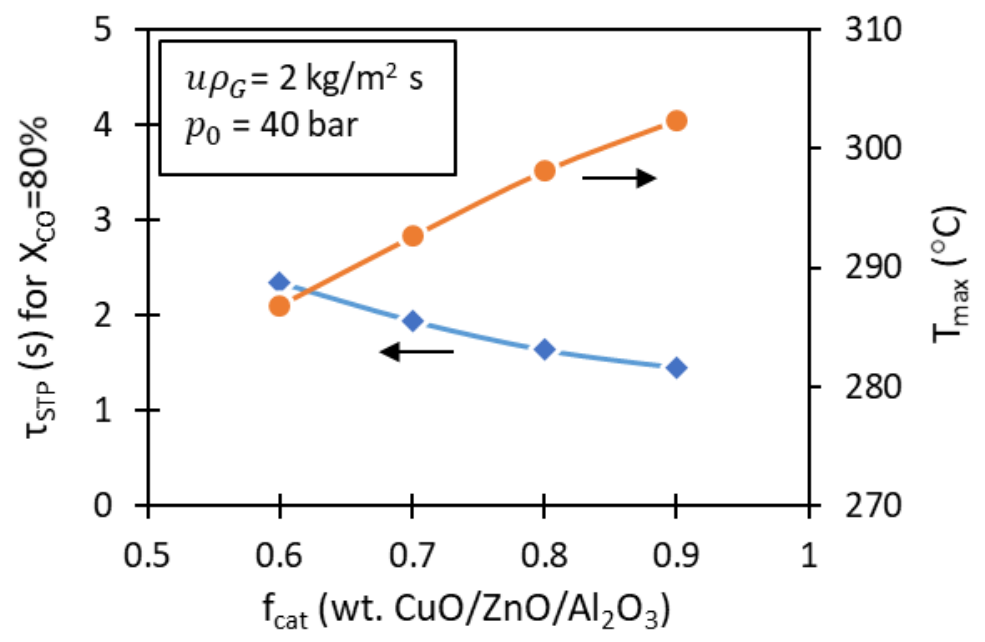

b)

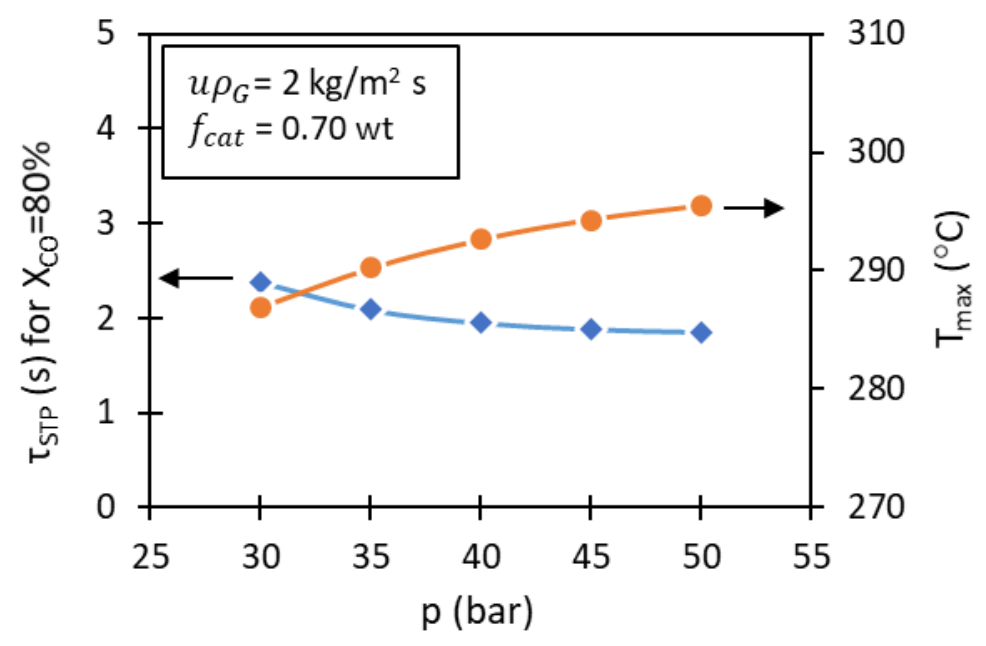

Figure 3. Parametric study: influence of mass flux (a), fraction of catalyst (b) and pressure (c) on the space time necessary for $80 \%$ CO conversion. Constant conditions: $\boldsymbol{T}_{\mathbf{0}}=250^{\circ} \mathrm{C}, \boldsymbol{\Delta} \boldsymbol{T}_{\boldsymbol{w}}=$ 0 , and $\boldsymbol{D}_{\boldsymbol{R}}=0.03 \mathrm{~m}$. 


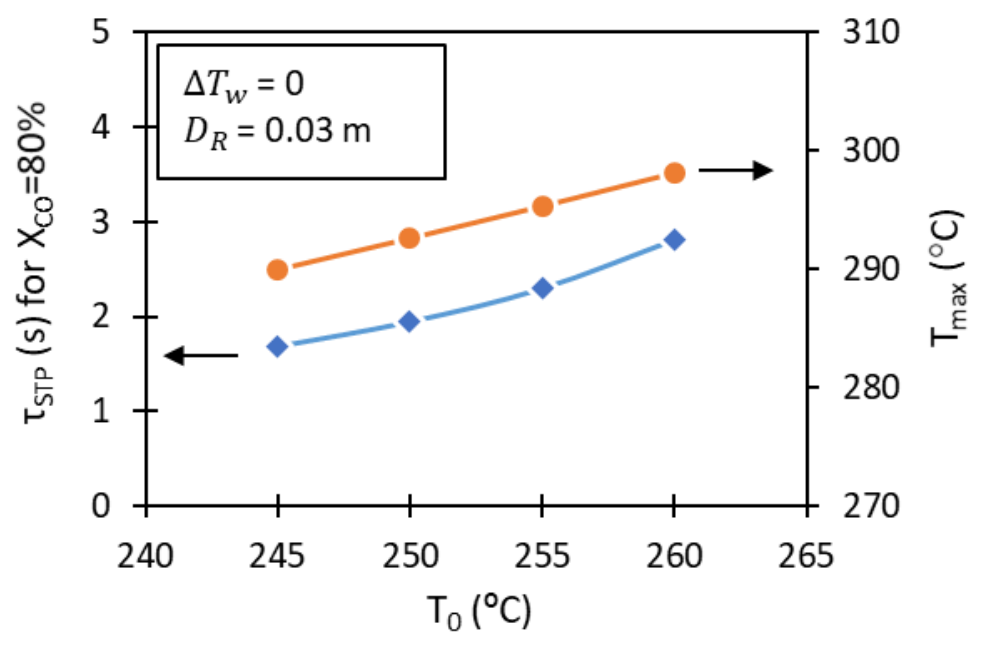

a)

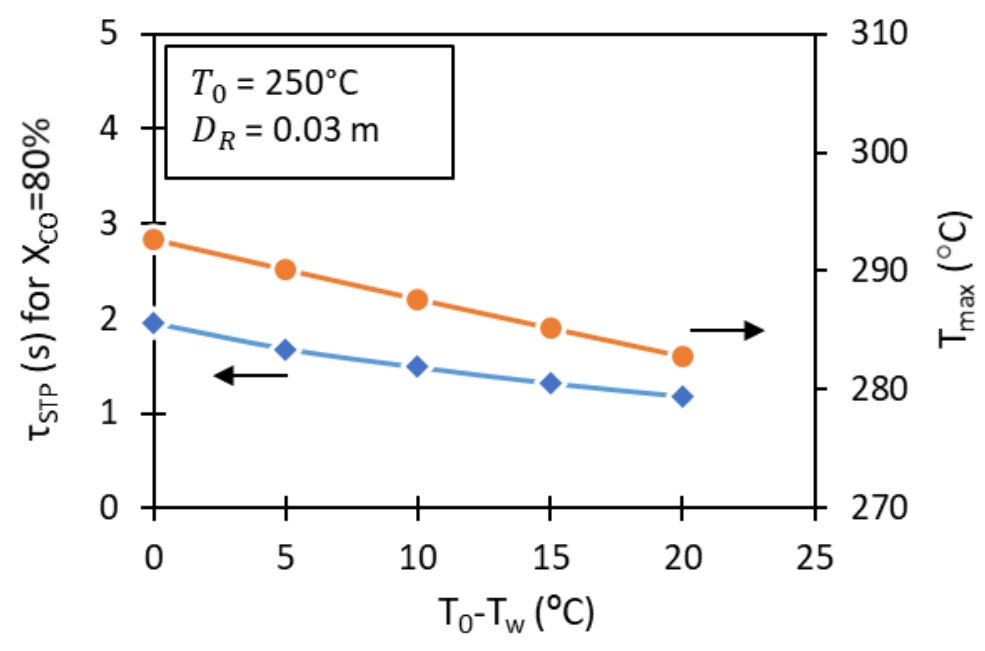

b)

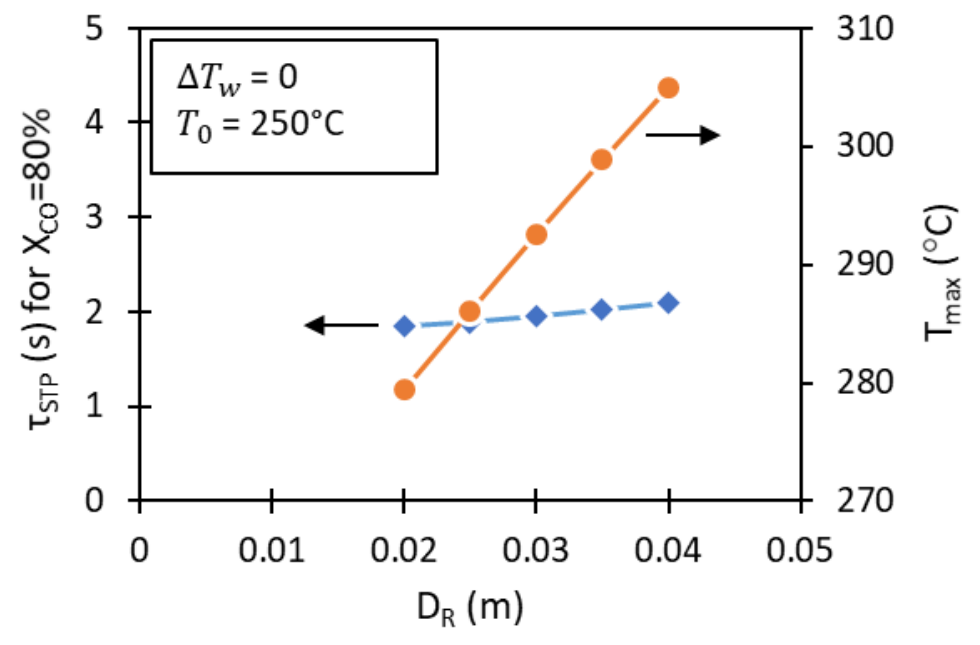

Figure 4. Parametric study: influence of feed temperature (a), wall temperature (b) and reactor diameter (c) on the space time necessary for $80 \%$ CO conversion. Constant conditions: $u \rho_{G}=2 \mathrm{~kg} / \mathrm{m}^{2} \mathrm{~s}, p_{0}=40$ bar and $f_{c a t}=0.70 \mathrm{wt}$. 

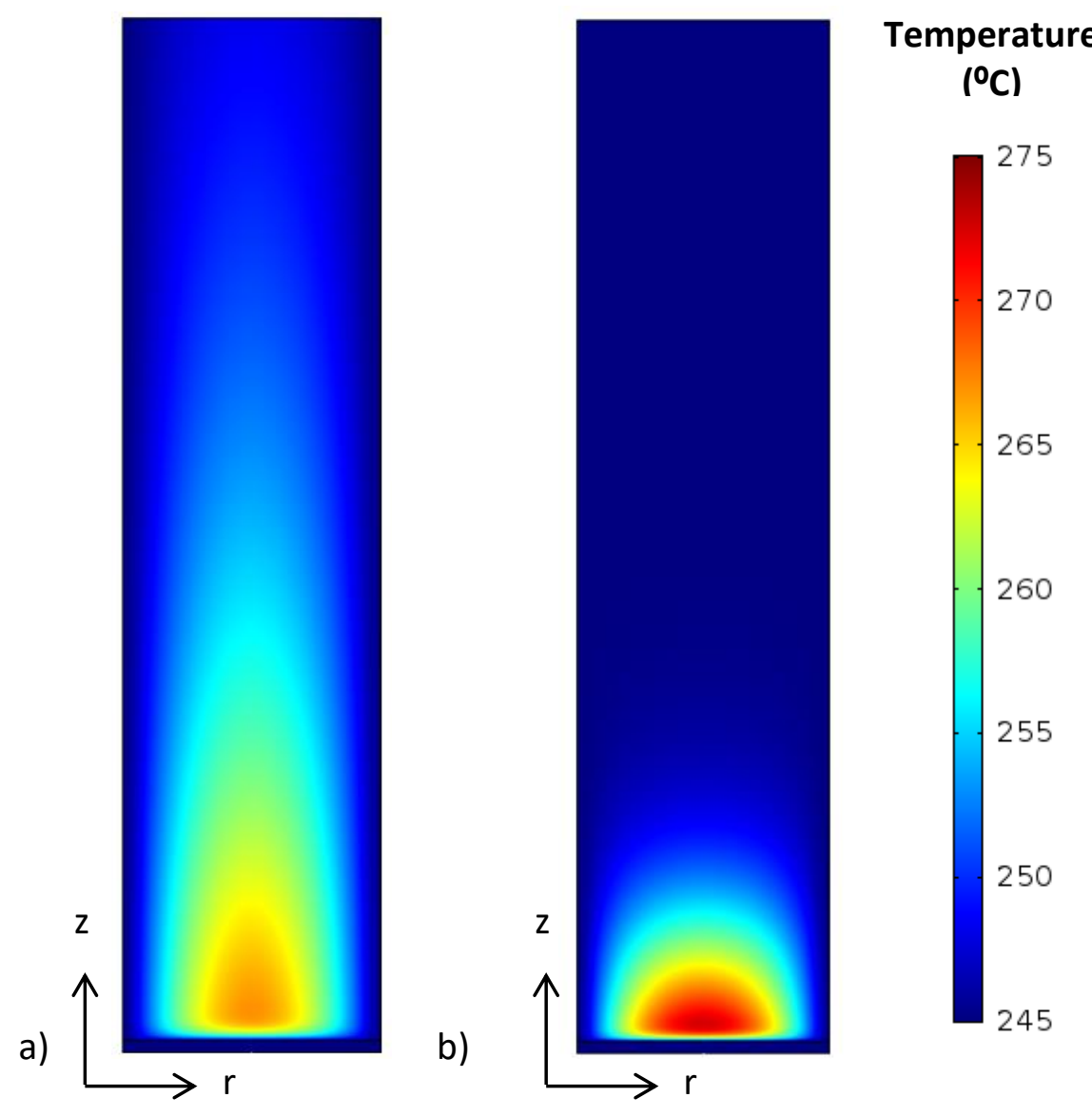

Figure 5. Simulation of the reactor model at the optimum operating conditions $\left(\boldsymbol{u} \rho_{G}=4\right.$ $\mathrm{kg} / \mathrm{m}^{2} \mathrm{~s}, \boldsymbol{T}_{\mathbf{0}}=245^{\circ} \mathrm{C}, \boldsymbol{\Delta} \boldsymbol{T}_{\boldsymbol{w}}=0, \boldsymbol{p}_{\mathbf{0}}=40 \mathrm{bar}, \boldsymbol{D}_{\boldsymbol{R}}=0.02 \mathrm{~m}$ and $\boldsymbol{L}_{\boldsymbol{R}}=8.50 \mathrm{~m}$ ): temperature gradient. Comparison of (a) direct DME synthesis $\left(\boldsymbol{f}_{\text {cat }}=0.70\right.$ wt.) and (b) methanol synthesis ( $\boldsymbol{f}_{\text {cat }}=1 \mathrm{wt}$.). 


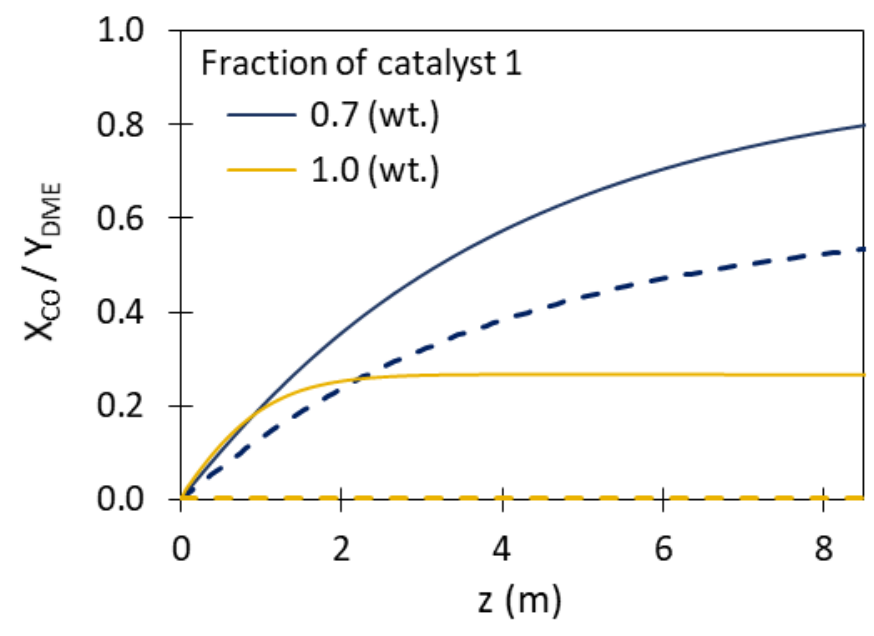

a)

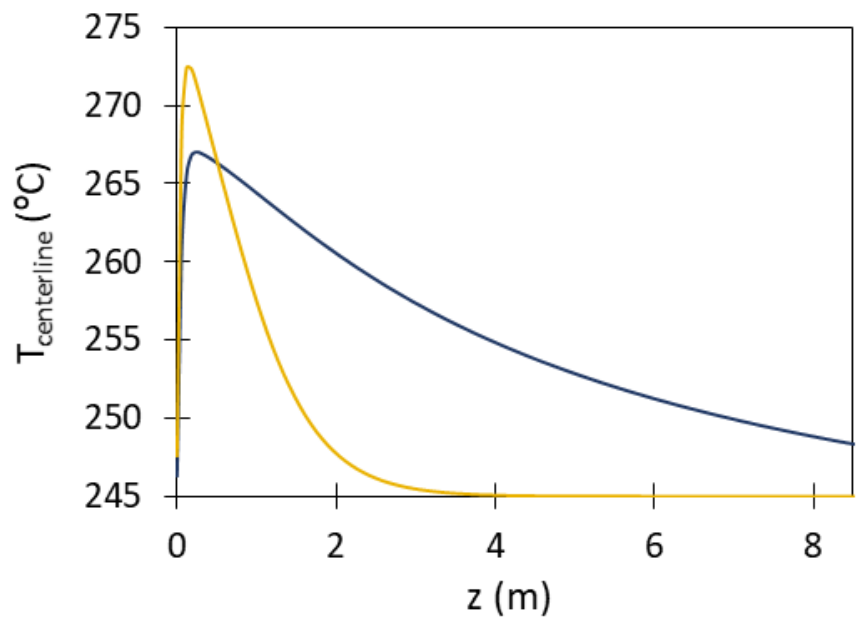

b)

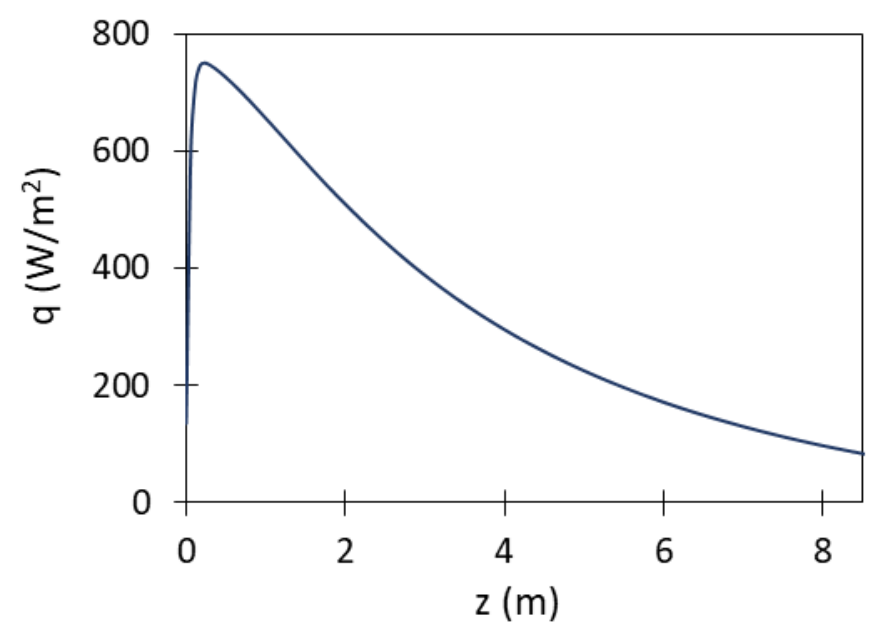

Figure 6. Simulation of the reactor model at the optimum operating conditions $\left(u \rho_{G}=4\right.$ $\mathrm{kg} / \mathrm{m}^{2} \mathrm{~s}, T_{0}=245^{\circ} \mathrm{C}, \Delta T_{w}=0, p_{0}=40 \mathrm{bar}, D_{R}=0.02 \mathrm{~m}$ and $L_{R}=8.50 \mathrm{~m}$ ): comparison of direct DME synthesis $\left(f_{\text {cat }}=0.70\right.$ wt. $)$ and methanol synthesis $\left(f_{\text {cat }}=1\right.$ wt.). (a) CO 
conversion (solid line) and DME yield (dashed line). (b) Centreline temperature. (c) Heat flux at the reactor wall $\left(r=D_{R} / 2=0.01 \mathrm{~m}\right)$ 


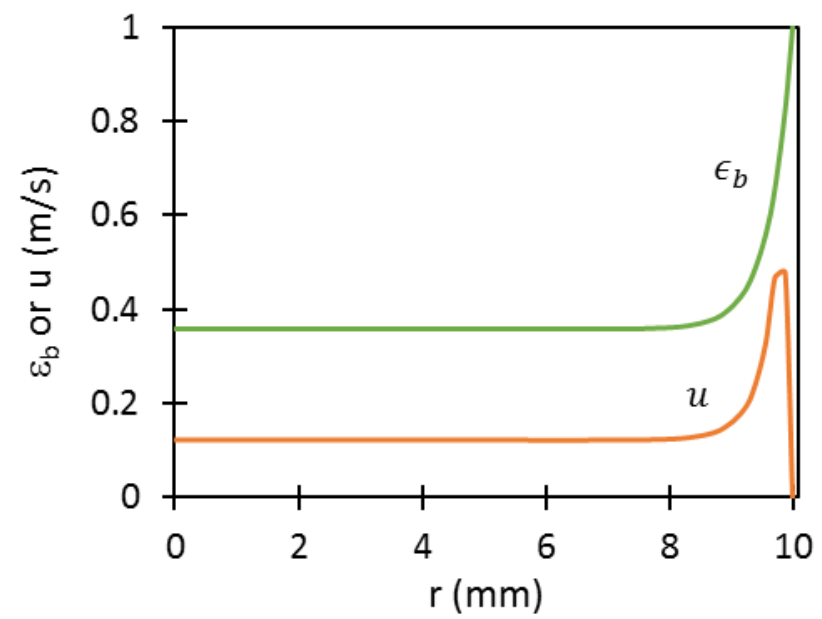

a)

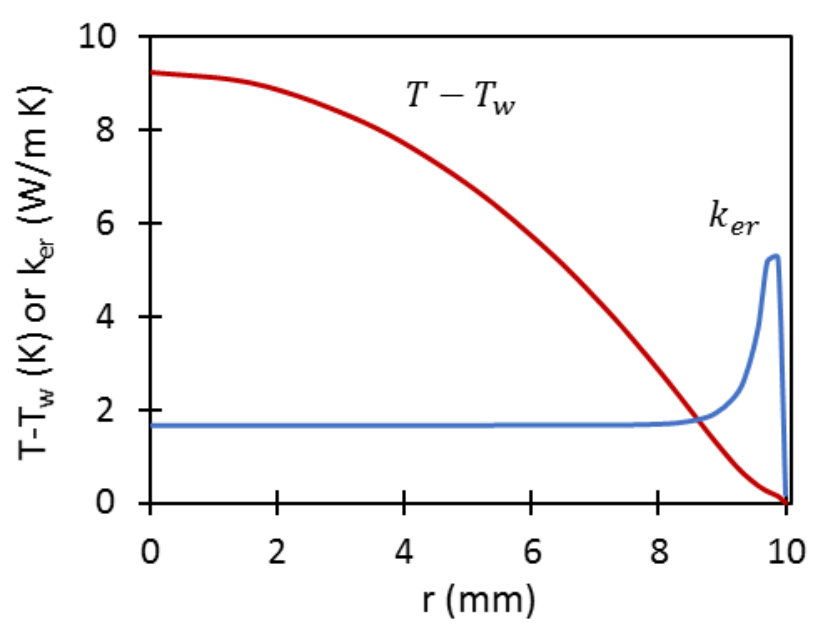

b)

Figure 7. Simulation of the reactor model at the optimized operating conditions $\left(\boldsymbol{u} \boldsymbol{\rho}_{G}=4\right.$ $\mathrm{kg} / \mathrm{m}^{2} \mathrm{~s}, \boldsymbol{T}_{\mathbf{0}}=245^{\circ} \mathrm{C}, \boldsymbol{\Delta} \boldsymbol{T}_{\boldsymbol{w}}=0, \boldsymbol{p}_{\mathbf{0}}=40 \mathrm{bar}, \boldsymbol{D}_{\boldsymbol{R}}=0.02 \mathrm{~m}$ and $\boldsymbol{L}_{\boldsymbol{R}}=8.50 \mathrm{~m}$ ): radial profiles at z $=\boldsymbol{L}_{\boldsymbol{R}} / 2=4.25 \mathrm{~m}$ of (a) bed porosity and superficial velocity and (b) radial temperature profile and radial thermal conductivity. 